\title{
Nanotinten für den Inkjet-Druck biomedizinischer Sensorkomponenten
}

\author{
M. Fritsch ${ }^{{ }_{1} 1}$, S. Mosch ${ }^{* 1}$, N. Trofimenko ${ }^{* 1}$, V. Sauchuk ${ }^{{ }_{1} 1}$, M. Vinnichenko $0^{* 1}$, \\ M. Kusnezoff ${ }^{* 1}, N$. Beshchasna ${ }^{* 2}, M$. Draz ${ }^{* 2}$, L. Röhmhildt $^{* 2}$, J. Opitz $^{* 2}$ \\ ${ }^{1}$ Fraunhofer IKTS Institut, Abt. Materialien und Komponenten, Dresden, Deutschland \\ ${ }^{2}$ Fraunhofer IKTS Institut, Abt. Bio- und Nanotechnologie, Dresden, Deutschland \\ Kontakt:marco.fritsch@ikts.fraunhofer.de
}

\section{Einleitung}

Die rasante Entwicklung biomedizinischer Sensoren führt zu zahlreichen neuen Anwendungen in den Bereichen Point-of-Care, Lab/Organ-on Chip, Umwelt- und Wellnesssensoren. Hierfür sind insbesondere dünne und mechanisch flexible Substratmaterialien, wie beispielsweise Polymerfolien und Papier, attraktiv für eine unscheinbare Integration von Sensoren für körpernahe Anwendungen bei einem vergleichsweise geringen Preis. Für Sensoren, die auf den Anwender stark individualisiert werden sollen (u.a. Berücksichtigung anwenderspezifischer Merkmale), weisen konventionelle mikroelektronische Herstellungsmethoden wie Fotolithografie Nachteile bezüglich der Möglichkeit eines schnellen Designwechsels und der damit einhergehenden Herstellungskosten für kleinere bis kleinste Stückzahlen auf. Aus diesem Grund werden digitale und additive Direktdruckverfahren für Funktionsmaterialien seit einigen Jahren entwickelt und erprobt. Die Kombination solcher Druckverfahren mit flexiblen Substratmaterialien im Rollenformat bietet zukünftig die Möglichkeit einer skalierbaren Massenproduktion von Sensoren in der Rolle-zu-Rolle Herstellung (R2R). Insbesondere der Inkjetdruck bietet hier als digitales Druckverfahren, im Vergleich zu anderen Druckmethoden, eine einfache, kostensparende Individualisierung des Druckbildes (kein Verbrauch an Sieben oder von Gravurzylindern) bei gleichzeitiger Skalierbarkeit der Druckfläche (modulare Integration von Druckköpfen mit mehreren Tausend Druckdüsen). Ein bislang wesentliches Problem zur technischen Umsetzung dieses Konzeptes liegt an der begrenzten Verfügbarkeit kompatibler Funktionsmaterialien in Form von Materialtinten. Zum einen weisen die adressierten preiswerten Polymerfolien (z.B. PET) sowie Papiersubstrate eine deutlich begrenzte thermische Stabilität von kleiner $200{ }^{\circ} \mathrm{C}$ auf. Eine Ausnahme bilden hier spezielle Thermoplastmaterialien, wie Polyimid, welche bis ca. $350{ }^{\circ} \mathrm{C}$ thermisch stabil, aber aufgrund ihres hohen Materialpreises für viele Sensorapplikationen nicht attraktiv sind. Zum anderen fordert der Inkjet-Druck eine Begrenzung der maximalen Partikelgröße in den Materialtinten von kleiner 0,5 bis $1,0 \mu \mathrm{m}$ (Vermeidung Zusetzen der kleinen Druckdüsen), sodass viele Ausgangsrohstoffe in Form bekannter Materialpulver nicht direkt verwendet werden können. Aus diesem Grund entwickelt das Fraunhofer IKTS spezielle Nanotinten, mit denen mittels Inkjet-Druck metallische Elektroden und Sensorkomponenten (u.a. Aktivmaterialien) auf thermisch empfindlichen Substraten abgeschieden werden. Hierbei sind insbesondere die entwickelten Gold- und Platintinten attraktiv für die Herstellung gedruckter Komponenten für
Biosensoren, da diese Druckschichten im Gegensatz zu Silber kompatibel zu Zellkulturen und Bioanalyten sind. Mit innen kann man Elektroden drucken, die mit biosensitiven Schichten weiter funktionalisiert werden können (Bsp. Aptamer-basierte Reaktionen [1]). Gedruckte PlatinSchichten wurden auch als pH-Sensor, Mikroheizer und Elektroden für flexible Sensoren evaluiert [2-5]. Gedruckte Silbermetallisierungen werden hingegen meist für die elektrische Kontaktierung genutzt, sind aber auch zur Realisierung von pseudo Referenzelektroden durch Funktionalisierung $\mathrm{zu} \mathrm{Ag/AgCl}$ oder als Temperatursensor [6-7] nutzbar

\section{Methoden und Materialien}

Silber-, Gold- und Platin-Nanopartikel wurden mittels nasschemischer Fällungsreaktionen erzeugt und für die Formulierung von Drucktinten mit 20 m.- $\%$ und 30 m.- $\%$ Feststoffgehalt genutzt (Beschreibung Synthese siehe [2, 3]. Die Tinten wurden hinsichtlich Viskosität (Haake Rheo Stress RS 150), Oberflächenspannung (Krüss, DSA100), Partikelgrößenverteilung und Sedimentationsstabilität (LUMiSizer®, LUM GmbH) so eingestellt, dass sie mit gängigen Inkjet-Druckköpfen kompatibel sind. Die Druckversuche wurden mit einem Dimatix DMP Material Printer mit 10 pL Druckköpfen auf Papier- und PET-Folien durchgeführt. Die Druckschichten wurden in einem Trockenschrank bei $80{ }^{\circ} \mathrm{C}$ getrocknet und folgend im Bereich 150 ${ }^{\circ} \mathrm{C}$ bis $200{ }^{\circ} \mathrm{C}$ gesintert. Alternativ wurde eine schnelle Sinterung mittel Linienlaser im Millisekundenbereich (LI$\mathrm{MO} \mathrm{GmbH}$ ) evaluiert. Die gesinterten Druckschichten wurden hinsichtlich elektrischem Widerstand mittels 4Punkt-Messung sowie Charakterisierung des Gefüges unter Nutzung der Rasterelektronenmikroskopie charakterisiert (NVision 40 Zeiss). Eine Biofunktionalisierung von Goldschichten wurde für die Detektion von $\alpha$-Thrombin, welches ein bekanntes Modellsystem für Bioanalyte ist, durchgeführt. Hierbei wurde die Goldoberfläche mit Aptamer und Methylenblau (elektrochemischer Redoxindikator) funktionalisiert und mittels elektrochemischer Analyse bewertet.

\section{Ergebnisse und Diskussion}

Die Abb.1 zeigt die hergestellten Partikel der verschiedenen Nanotinten. Die mittleren Partikelgrößen für $\mathrm{Ag}, \mathrm{Au}$ und Pt Nanotinten liegen deutlich < $200 \mathrm{~nm}$. Exemplarisch wurde auch eine Cu-Synthese erprobt. Die Tab.1 fasst wesentliche Tintenparameter zusammen. Die entwickelten Nanotinten weisen eine gute Sedimentationsstabilität auf 
und ließen sich reproduzierbar mit dem Inkjetsystem verdrucken.

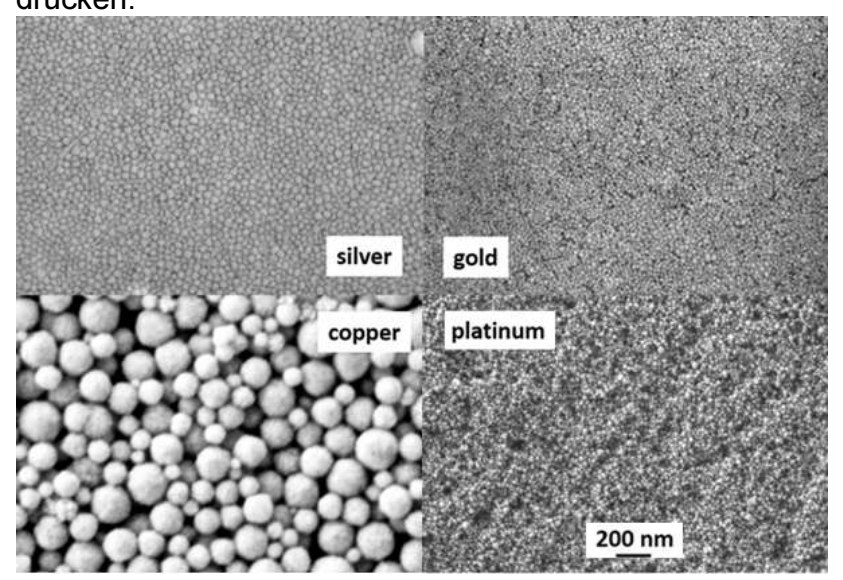

Abb. 1: Synthetisierte Partikel für Nanotinten

Tab. 1: Übersicht Tintenparameter

\begin{tabular}{|l|l|}
\hline Parameter & \multicolumn{1}{|c|}{ Nanotinten (Ag, Au und Pt) } \\
\hline Feststoffgehalt & 20 oder $30 \mathrm{~m} .-\%$ \\
\hline Dichte & 1,3 bis $1,5 \mathrm{~g} / \mathrm{cm}^{3}$ \\
\hline Oberflächenspannung & $30 \mathrm{bis} 35 \mathrm{mNm} @ 20^{\circ} \mathrm{C}$ \\
\hline Viskosität & $8-10 \mathrm{mPas} @ 100 / \mathrm{sec}$ and $22{ }^{\circ} \mathrm{C}$ \\
\hline Partikelgröße & $<200 \mathrm{~nm}$ \\
\hline Sedimentationsrate & $10^{-2} \mathrm{bis} 10^{-3} \mathrm{~mm} / \mathrm{Tag}$ \\
\hline
\end{tabular}

Die Tab. 2 fasst die erreichten elektrischen Widerstände der gedruckten und gesinterten Metallisierungen zusammen.

Tab. 2: Elektrischer Widerstand gedruckter Metallisierungen in $\mu \mathrm{Ohmcm}$ (x-fach bulk Wert ${ }^{* 1}$ )

\begin{tabular}{|l|l|l|l|}
\hline Parameter & \multicolumn{1}{|c|}{ Ag-LT-20 } & \multicolumn{1}{c|}{ Au-LT-20 } & \multicolumn{1}{c|}{ Pt-LT-20 } \\
\hline Sinterung & $150{ }^{\circ} \mathrm{C}$ & $200{ }^{\circ} \mathrm{C}$ & $200{ }^{\circ} \mathrm{C}$ \\
\hline PET-Folie & $11,1(2,5 \mathrm{x})$ & $32,9(5,3 \mathrm{x})$ & $1329(38 \mathrm{x})$ \\
\hline Papier & $8,5(2,0 \mathrm{x})$ & $34,6(5,6 \mathrm{x})$ & $303(9 \mathrm{x})$ \\
\hline Schichtdicke & $1,9 \mu \mathrm{m}$ & $0,9 \mu \mathrm{m}$ & $0,7 \mu \mathrm{m}$ \\
\hline${ }^{*} 1$ Abschätzung basierend auf Filmkontur mit Füllfaktor $60 \%$ \\
und Porosität 40\%. \\
\hline
\end{tabular}

Die erreichten Widerstände, insbesondere bei $\mathrm{Ag}$ und $\mathrm{Au}$, liegen im Bereich des zwei- bis sechsfachen Bulkwiderstands, was für die meisten sensorischen Anwendungen ausreichend hoch sein sollte. Auch die gedruckten PtSchichten weisen, bei vergleichsweise sehr niedrigen Sintertemperaturen von $200{ }^{\circ} \mathrm{C}$, eine relativ gute Leitfähigkeit auf. Die alternative und schnelle Sintermethode mittels Diodenlasersinterung führte auch zu einer Sinterung der Druckschichten und Widerstände die einem ähnlichen Niveau derer der Ofensinterung entsprechen. Die Schichtdicken nach der Sinterung liegen im Bereich 0,7 bis ca. 2 $\mu \mathrm{m}$, was auch durch Variation der Anzahl von Drucklagen anwendungsspezifisch angepasst werden kann. Die Abb.2 verdeutlicht die mechanische Flexibilität der hergestellten Metallisierungen.
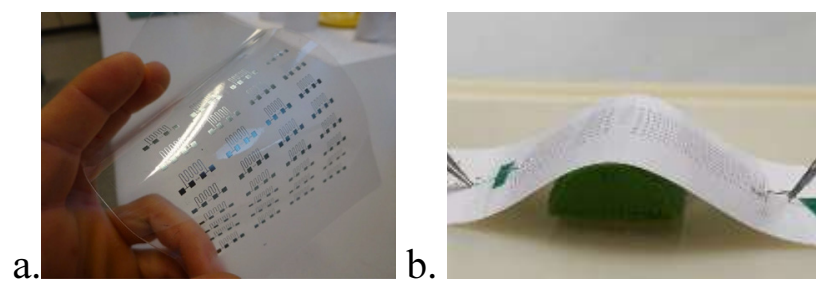

Abb. 2: Inkjetgedruckte und mechanisch flexible Metallisierungen auf (a) PET-Folie und (b) Papier

Die Funktionalisierung gedruckter Goldelektroden zur Detektion von $\alpha$-Thrombin wurde auf Basis einer AptamerBeschichtung mit Methylenblau (Prinzip siehe Abb. 3) durchgeführt.

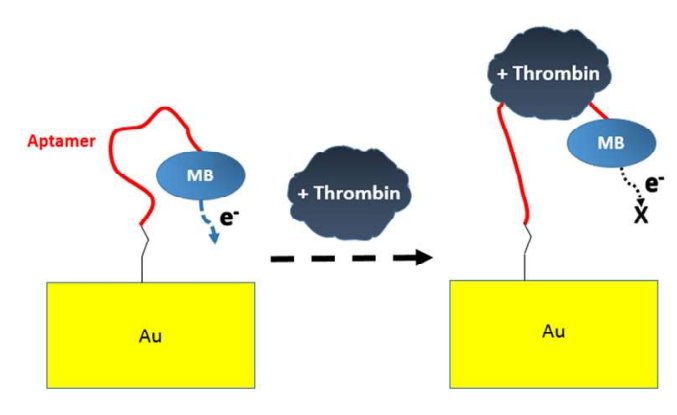

Abb. 3: Prinzip elektrochemische Detektion von $\alpha$ Thrombin auf Basis funktionalisierter Goldelektroden

Die entwickelten Elektroden konnten erfolgreich elektrochemisch ausgewertet werden. Es wurde eine reversible Redoxreaktion für die $\alpha$-Thrombin Detektion im Spannungsbereich von $-0,1$ bis $-0,4 \mathrm{~V}$ beobachtet (Abb.4).

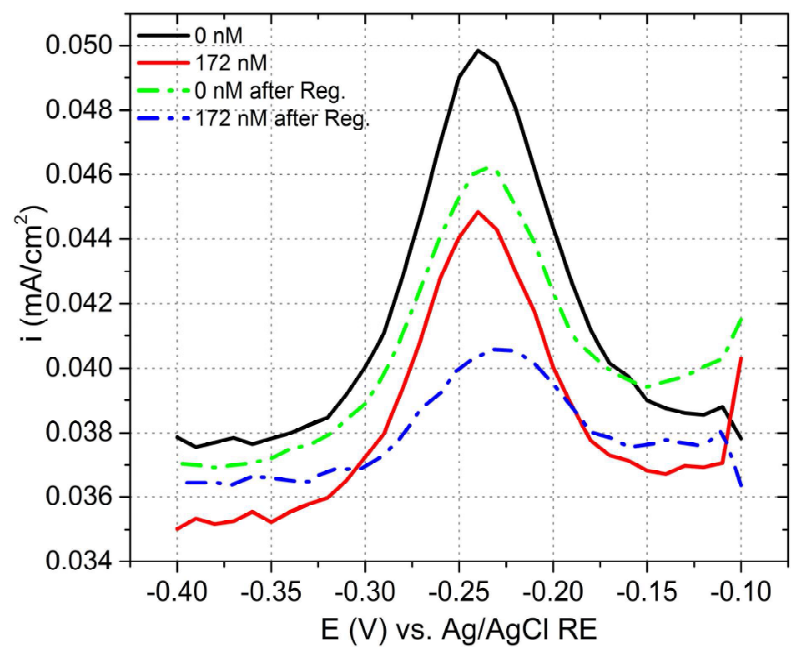

Abb. 4: Elektrochemische Auswertung zur $\alpha$-Thrombin Detektion funktionalisierter Goldelektroden

Auf Basis der erreichten Ergebnisse werden verschiedene Biosensorkonzepte verfolgt. Hierbei wird insbesondere eine Kombination verschiedener Sensoren (Bestimmung Temperatur, $\mathrm{pH}$ und elektrochemische Detektion von Ana- 
lyten) auf einer miniaturisierten und mechanisch flexiblen „Sensorplattform" als attraktiv angesehen.

\section{Literatur}

[1] DRAZ, M.S.: Aptamer-based Electrochemical Biosensing on Sensor Chips with Printed Gold Electrodes, Masterarbeit, Masterarbeit, TU-Dresden, 2017

[2] JURK, R.; MOSCH, S.; FRITSCH, M.; IHLE, M.: Synthesis of nano metal particles for low sintering conductive inks, Fraunhofer Direct Digital Manufacturing Conference 2014 - DDMC, Berlin (12./13.3.2014), E-ISBN: 978-3-8396-9128-1 , pp.269-273, 2014

[3] ZEA, M.; MOYA, A.; FRITSCH, M.; ROMAN, E.; VILLA, R.; GABRIEL, G.: "Enhanced performance stability of iridium oxide based $\mathrm{pH}$ sensors fabricated on rough inkjet-printed platinum", ACS Appl. Mater. Interfaces 2019, 11, pp.15160-15169

[4] KHAN, S.; NGUYEN, T.P.; LUBEJ, M.; THIERY, L.; VAIRAC, P.; BRIAND, D.: Low-power printed microhotplates through aerosol jetting of gold on thin polyimide membranes, Microelectronic Engineering, 194, 2018, pp.71-78

[5] SCHUBERT, M.; WANG, Y.; Fritsch, M.; VINNICHENKO, M.; REBOHLE, L.; SCHUMANN, T.; BOCK, K.: Evaluation of Nanoparticle Inks on Flexible and Stretchable Substrates for Biocompatible Application, 7th Electronic System-Integration Technology Conference (ESTC) 2018, 18-21.September 2018 Dresden, proceeding IEEE, 2018. https://ieeexplore.ieee.org/document/8546494

[6] VINNICHENKO, M.; MAKAROV, D.; FRITSCH, M.; VOITSEKHIVSKA, T.; SAUCHUK, V.; KUSNEZOFF, M.: Realizing Flexible High-Performance Silver Interconnects on Thin and Ultrathin Substrates by InkjetPrinting and Innovative Laser Treatment, 14th International Conference on Modern Materials and Technologies (CIMTEC), 04-14.06.2018, Perugia Italy, 2018

[7] Moya, A.; ORTEGA-RIBERA, M.; GUIMERA, X.; SOWADE, E.; ZEA, M.; ILLA, X.; RAMON, E.; VILLA, R.; GRACIA-SANCHO, J.; GABRIEL, G.: Online oxygen monitoring using integrated inkjet-printed sensors in a liver-on-a-chip system, Lab Chip, 18, 2018, pp.2023-2035

\section{Danksagung}

Die vorgestellten Ergebnisse wurden zum Teil durch das Bundesministerium für Bildung und Forschung (BMBF) im Rahmen des Forschungsprojektes "DigiMan" (02P15B520) gefördert. 